\title{
PHASE TRANSITIONS AND DUALITY PROPERTIES OF A SUCCESSIONAL MODEL
}

\author{
NICOLAS LANCHIER, ${ }^{*}$ Université de Rouen
}

\begin{abstract}
The first purpose of this article is to study the phase transitions of a new interacting particle system. We consider two types of particle, each of which gives birth to particles of the same type as the parent. Particles of the second type can die, whereas those of the first type mutate into the second type. We prove that the three possible outcomes of the process, that is, extinction, survival of the type- $2 \mathrm{~s}$, or coexistence, may each occur, depending on the selected parameters. Our second, and main, objective, however, is to investigate the duality properties of the process; the corresponding dual process exhibits a structure somewhat different to that of well-known particle systems.
\end{abstract}

Keywords: Interacting particle system; competition model; phase transition; multitype contact process; forest fire model; duality

2000 Mathematics Subject Classification: Primary 60K35

\section{Introduction}

The spatial model we introduce in this paper is a continuous-time Markov process in which the state at time $t$ is a function $\xi_{t}: \mathbb{Z}^{d} \rightarrow\{0,1,2\}$. A site $x \in \mathbb{Z}^{d}$ is said to be empty at time $t$ if $\xi_{t}(x)=0$, and is said to be occupied by a particle of type 1 (a 'type-1') or of type 2 (a 'type-2') if $\xi_{t}(x)=1$ or $\xi_{t}(x)=2$, respectively. The evolution rules can be formulated as follows:

1. Each type-1 or type- 2 tries to give birth onto each of its neighbouring sites at rate $\lambda_{1}$ or $\lambda_{2}$, respectively. Here, the neighbours of a site $x \in \mathbb{Z}^{d}$ constitute the set of $y \in \mathbb{Z}^{d}$ such that $\|x-y\| \leq r$, where $\|\cdot\|$ is a norm and $r$ a positive constant.

2. If the offspring is to be sent to an empty site, the birth occurs. Otherwise, it is prevented.

3. Each type-1 becomes a type- 2 at rate 1 .

4. Each type- 2 dies, i.e. the state becomes 0 , at rate 1 .

If only type-2s are present, the system reduces to the contact process with parameter $\lambda_{2}$. In this case, there exists a critical value $\lambda_{\mathrm{c}} \in(0, \infty)$ such that the following holds: if $\lambda_{2} \leq \lambda_{\mathrm{c}}$ then the process converges in distribution to the all-empty state. Otherwise, there exists a stationary measure $\mu_{2}$ that concentrates on configurations with infinitely many type- $2 \mathrm{~s}-$ see, e.g. Liggett (1999). To avoid trivialities, we will assume from now on that $\xi_{0}$, i.e. the configuration at time 0 , contains infinitely many type- $1 \mathrm{~s}$ and type- $2 \mathrm{~s}$.

We first suppose that $\lambda_{1} \geq \lambda_{2}>\lambda_{\mathrm{c}}$ and consider two initial configurations $\xi_{0}^{1}$ and $\xi_{0}^{2}$, such that $\xi_{0}^{2}(x)=0$ if $\xi_{0}^{1}(x)=0$ and $\xi_{0}^{2}(x)=2$ otherwise, i.e. $\xi_{0}^{2}$ can be found from $\xi_{0}^{1}$ by replacing

Received 22 June 2004; revision received 27 September 2004.

* Postal address: Laboratoire de Mathématiques Raphaël Salem, UMR 6085, Université de Rouen, 76128 Mont Saint

Aignan, France. Email address: nicolas.lanchier@etu.univ-rouen.fr 
each type- 1 by a type-2. This makes $\xi_{t}^{2}$ a contact process with parameter $\lambda_{2}>\lambda_{\mathrm{c}}$. Then, relying on a standard argument from Harris (1972), we may run both processes on the same probability space in such a way that if $\xi_{t}^{2}(x)=2$ then $\xi_{t}^{1}(x) \neq 0$. This implies, in particular, that type-2s survive, i.e. that $\mathrm{P}\left(\right.$ for all $t \geq 0$, there exists $x$ such that $\left.\xi_{t}^{1}(x)=2\right)=1$.

We now suppose that $\lambda_{2}>\lambda_{1}>\lambda_{\mathrm{c}}$. In this case, due to a lack of monotonicity, the previous coupling fails. We then take $\xi_{0}^{1}=\xi_{0}^{2}$ and replace the transition $1 \rightarrow 2$ in $\xi_{t}^{1}$ by the transition $1 \rightarrow 0$ in $\xi_{t}^{2}$. This makes $\xi_{t}^{2}$ a multitype contact process with parameters $\lambda_{1}$ and $\lambda_{2}-$ see Neuhauser (1992). By running both processes on the same space, as before, we can prove that $\xi_{t}^{1}$ has more type-2s and fewer type-1s than $\xi_{t}^{2}$. Then, the results of Section 3 of Durrett and Neuhauser (1997) imply that $\xi_{t}^{1} \stackrel{\mathrm{w}}{\rightarrow} \mu_{2}$, where $\stackrel{\text { w }}{\rightarrow}$ ' denotes weak convergence. These results are summarized in the following theorem.

Theorem 1. We set $\lambda_{2}>\lambda_{\mathrm{c}}$.

1. If $\lambda_{1} \geq \lambda_{2}$ then type-2s survive, i.e.

$$
\mathrm{P}\left(\text { for all } t \geq 0 \text {, there exists } x \text { such that } \xi_{t}(x)=2\right)=1 \text {. }
$$

2. If $\lambda_{1}<\lambda_{2}$ then type-2s win, i.e. $\xi_{t} \stackrel{\mathrm{W}}{\rightarrow} \mu_{2}$, the upper invariant measure of the contact process.

We now take $\lambda_{2}$ to be close to 0 . If we suppress the transition $0 \rightarrow 2$, i.e. if $\lambda_{2}=0$, we can view the process as a forest fire model, which translates as $0 \equiv$ alive, $1 \equiv$ on fire, and 2 三 burnt. By using a rescaling argument, Durrett and Neuhauser (1991) proved that, in the two-dimensional nearest-neighbour case, the process exhibits a phase transition. More precisely, there is a critical value $\alpha_{\mathrm{c}} \in(0, \infty)$ such that, if $\lambda_{1}>\alpha_{\mathrm{c}}$, there exists a nontrivial stationary measure $v$ that concentrates on configurations with infinitely many type-1s and type-2s. Theorem 2 tells us that type- $1 \mathrm{~s}$ and type- $2 \mathrm{~s}$ still coexist, for sufficiently small $\lambda_{2}>0$.

Theorem 2. We set $d=2$ and $\lambda_{1}>\alpha_{\mathrm{c}}$. There exists a $\beta_{\mathrm{c}} \in(0, \infty)$ such that, if $\lambda_{2} \leq \beta_{\mathrm{c}}$, type- 1 s and type- 2 s coexist, i.e. $\xi_{t} \stackrel{\mathrm{W}}{\rightarrow} v$ with $v\left(\xi_{t}(x)=1\right) \neq 0$.

We now come to the main result of this paper: to improve Theorem 1 by proving that type- $2 \mathrm{~s}$ still win if $\lambda_{1}=\lambda_{2}$. Although Theorem 3 is not surprising, the proof relies on a rather interesting property of the dual process.

Theorem 3. If $\lambda_{1}=\lambda_{2}>\lambda_{\mathrm{c}}$ and $\xi_{0}$ is translation invariant, then type- 2 s win, i.e. $\xi_{t} \stackrel{\mathrm{w}}{\rightarrow} \mu_{2}$.

The main ingredient of the proof is duality. To deduce the structure of the dual process, we will start by focusing on the multitype contact process, which we denote by $\eta_{t}: \mathbb{Z}^{d} \rightarrow\{0,1,2\}$. Then we will show how to construct both processes $\xi_{t}$ and $\eta_{t}$ using a graphical representation, and see what it is that makes the dual process of $\xi_{t}$ different from that of the multitype contact process. First consider $\eta_{t}$ : we fix $\lambda_{1}=\lambda_{2}$, denote by $\lambda$ their common value, and, for $x, y \in \mathbb{Z}^{d},\|x-y\| \leq r$, let $\left\{T_{n}^{x, y}: n \geq 1\right\}$ and $\left\{U_{n}^{x}: n \geq 1\right\}$ be the arrival times of Poisson processes with rates $\lambda$ and 1 , respectively. At times $T_{n}^{x, y}$, we (figuratively speaking) draw an arrow from site $x$ to site $y$ while, at times $U_{n}^{x}$, we put a cross at site $x$. This may be interpreted as follows: if, at time $T_{n}^{x, y}$, the site $x$ is occupied and the site $y$ empty, then the particle at $x$ gives birth to a particle of the same type at $y$. At time $U_{n}^{x}$, we remove the particle at $x$, if it is present. These evolution rules define $\eta_{t}$ as the multitype contact process with parameters $\lambda_{1}=\lambda_{2}=\lambda$. (See Neuhauser (1992) for a complete study of this process.) Finally, we say that there is a path 
from $(y, 0)$ to $(x, t)$ if there is a sequence of times $s_{0}=0<s_{1}<\cdots<s_{n+1}=t$, and one of spatial locations $x_{0}=y, x_{1}, \ldots, x_{n}=x$, such that the following two conditions hold:

1. For $i=1,2, \ldots, n$, there is an arrow from $x_{i-1}$ to $x_{i}$ at time $s_{i}$.

2. For $i=0,1, \ldots, n$, the vertical segments $\left\{x_{i}\right\} \times\left(s_{i}, s_{i+1}\right)$ do not contain any crosses.

Since the crosses kill both types of particle, the ancestor of a particle at $x$ at time $t$ can be located at $y$ at time 0 only if there is a path from $(y, 0)$ to $(x, t)$. In conclusion, the dual process starting at $(x, t)$ - that is, the set of the potential ancestors of site $x$ at time $t$-will be defined by reversing the arrows and letting

$$
\tilde{\eta}_{s}^{(x, t)}=\left\{y \in \mathbb{Z}^{d}: \text { there is a path from }(x, \tilde{0}) \text { to }(y, \tilde{s})\right\},
$$

where $\tilde{s}=t-s$ (and $\tilde{0} \equiv t$ ). From a topological point of view, it is known that the set $\left\{\tilde{\eta}_{s}^{(x, t)}: s \leq t\right\}$ exhibits a tree structure - see, for instance, Durrett (1995), Liggett (1999), or Neuhauser (1992).

A natural way to construct our successional model would be to introduce a new collection of Poisson processes $\left\{V_{n}^{x}: n \geq 1\right\}, x \in \mathbb{Z}^{d}$, with rate 1 , put a dot at site $x$ at times $\left\{V_{n}^{x}: n \geq 1\right\}$, and pretend that the effect of a cross is now to kill particles of type 2 only, while that of a dot is to 'paint' particles of type 1 the 'colour' 2 . The first ingredient in proving Theorem 3 is to observe that, since the particles of type 1 do not see the crosses, and those of type 2 do not see the dots, we can superimpose the crosses and the dots. In other words, we use the same collections of Poisson processes as in the multitype contact process. That is, we remove the dots and say that if, at time $U_{n}^{x}$, the site $x$ is occupied by a particle of type 1 or of type 2 , then we paint this particle the colour 2 or, respectively, we kill it. Relying on this graphical representation, it is natural to extend the definition of a path by replacing condition 2 , above, as follows:

$2^{\prime}$. The set $\bigcup_{i=0}^{n}\left\{x_{i}\right\} \times\left(s_{i}, s_{i+1}\right)$ contains at most one cross.

That is, a path is now allowed to contain at most one cross. In view of the crosses' effect, if there is a path from $(y, 0)$ to $(x, t)$, then a particle of type 1 at $y$ at time 0 can certainly be the ancestor of a particle of type 2 located at $x$ at time $t$ if it goes through one cross on its way to $(x, t)$. If we define the dual process starting at $(x, t)$ by letting

$$
\tilde{\xi}_{s}^{(x, t)}=\left\{y \in \mathbb{Z}^{d}: \text { there is a path from }(x, \tilde{0}) \text { to }(y, \tilde{s})\right\},
$$

the set $\left\{\tilde{\xi}_{s}^{(x, t)}: s \leq t\right\}$ now exhibits a tree structure divided into two stages, depending on the number of crosses ( 0 or 1$)$ an ancestor encounters on the way to $(x, t)$. Then, a site $y \in \mathbb{Z}^{d}$ will be called an upper ancestor or a lower ancestor if there is a path from $(y, 0)$ to $(x, t)$ containing 0 or 1 cross, respectively. We will use the terminology upper or lower stage to refer to the set of the upper or lower ancestors. Here, as in the multitype contact process, the tree structure of the dual process allows us to define an ancestor hierarchy, in which the members are arranged according to the order in which they determine the colour of $(x, t)$. The first ancestor in the hierarchy will be called the determining particle, and the first upper ancestor the distinguished particle.

With this construction in mind, we can sketch the proof of Theorem 3 as follows. The first step is to prove that the last time $\Theta_{1}$ at which the determining particle belongs to the upper stage of the tree structure is almost surely finite, i.e. $\mathrm{P}\left(\Theta_{1}=\infty\right)=0$. The rest of the proof then relies on the observation that, once trapped inside the lower stage, the determining particle can 
only paint $(x, t)$ the colour 2 , since the crosses' effect is to change the colour of the type- $1 \mathrm{~s}$. In $d \leq 2$, we wait until time $\Theta_{1}$ and then prove that the distinguished particle and the determining particle coalesce with probability 1 , so that they will land, for $t$ sufficiently large, on the same site. If this site is initially occupied by a particle of type 1 , the determining particle will paint $(x, t)$ the colour 2. If the site is occupied by a particle of type 2 , the distinguished particle will paint $(x, t)$ the colour 2 unless a lower ancestor does so earlier. In $d \geq 3$, we will rely on the fact that $\Theta_{1}<\infty$ to construct inductively a sequence of lower ancestors $\zeta_{s}(k)$ that come before the distinguished particle in the ancestor hierarchy. We will then extract a subsequence of candidates $\zeta_{s}\left(k_{i}\right)$ that do not coalesce, and we will conclude that at least one lands on a type-1 and paints $(x, t)$ the colour 2 .

Barring Section 2, where we focus on Theorem 2, the rest of the article is devoted to the proof of Theorem 3. In Section 3, we investigate in greater detail the duality properties of the process. Relying on the construction given in Section 3, we then prove Theorem 3 in two steps: in Section 4 , we start by dealing with the case $d \leq 2$, and then conclude, in Section 5 , with the case $d \geq 3$.

\section{Proof of Theorem 2}

This section is devoted to the proof of Theorem 2. In particular, we will prove that, in dimension two, coexistence occurs for an open set of values $\left(\lambda_{1}, \lambda_{2}\right)$ in $\mathbb{R}^{2}$. We conjecture that such a property holds in any dimension, but our proof heavily relies on Lemma 1.1 of Durrett and Neuhauser (1991), which has been proved in $d=2$ only.

If $\lambda_{2}=0$, we recall that 0 can be interpreted as a living tree, 1 as a burning tree, and 2 as a burnt site. We let $B=(-L, L)^{2}$, where $L$ is a positive integer, and, for any $m \in \mathbb{Z}$, let $B_{m}=m L e_{1}+B$, where $e_{1}=(1,0)$ is the first unit vector. For $(m, n) \in \mathbb{Z}^{2}$ with $m$ and $n$ both even, or $m$ and $n$ both odd, we say that $(m, n)$ is occupied if the following two conditions are satisfied.

1. There are more than $L^{1 / 2}$ burning trees in $B_{m}$ at some time $t \in[n \gamma L,(n+1) \gamma L]$.

2. There is at least one burning tree in $B_{m}$ at all times $t \in[(n+1) \gamma L,(n+2) \gamma L]$.

Here, $\gamma$ is a positive constant. The following lemma implies that there exists a critical value $\alpha_{\mathrm{c}} \in(0, \infty)$ such that coexistence occurs for $\lambda_{1}>\alpha_{\mathrm{c}}$ and $\lambda_{2}=0$.

Lemma 1. (Lemma 1.1 of Durrett and Neuhauser (1991).) There exists $\alpha_{\mathrm{c}} \in(0, \infty)$ such that, if $\lambda_{1}>\alpha_{\mathrm{c}}$ and $\lambda_{2}=0, \gamma$ and $L$ can be chosen so that the set of occupied sites dominates the set of wet sites in a one-dependent oriented percolation process with parameter $p=1-6^{-36}$.

Given $\gamma$ and $L$, it is clear that there exists $\beta_{\mathrm{c}} \in(0, \infty)$ such that, if $\lambda_{2} \leq \beta_{\mathrm{c}}$, the probability that the type-2s give birth in the spacetime box $B_{m} \times[n \gamma L,(n+2) \gamma L]$ can be bounded by $6^{-36}$. This implies that Lemma 1 holds for $\lambda_{2} \leq \beta_{\mathrm{c}}$ and $p=1-2 \times 6^{-36}$. In particular, there exists, for $\lambda_{1}>\alpha_{\mathrm{c}}$ and $\lambda_{2} \leq \beta_{\mathrm{c}}$, an infinite cluster of occupied sites - see Section 10 of Durrett (1984). In conclusion, there is a stationary distribution that concentrates on configurations with infinitely many type-1s and type-2s - see, e.g. Section 4 of Durrett (1995). This completes the proof of Theorem 2.

\section{Construction and properties of the dual process}

From now on, and until the end of the paper, we suppose that $\lambda_{1}=\lambda_{2}$ and denote by $\lambda$ their common value. We start by constructing the process from a collection of Poisson processes in 


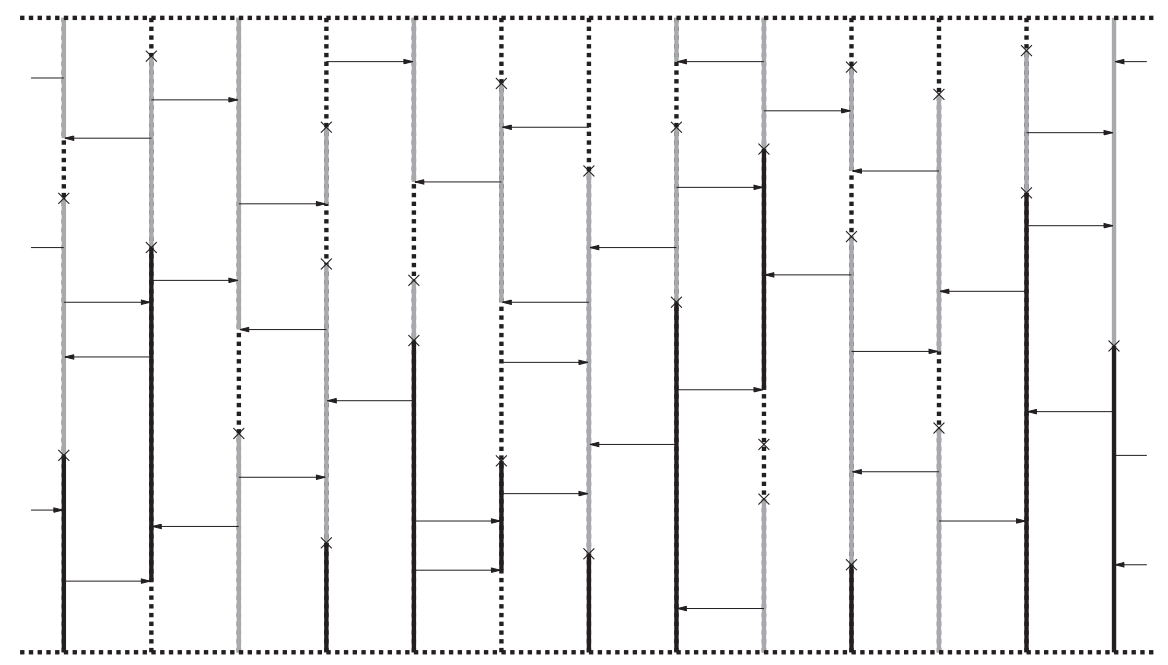

FIGURE 1: Harris's graphical representation. The black lines refer to type-1s, the grey lines to type-2s, and the dotted lines to empty sites.

the following way (as was discussed above). For $x, y \in \mathbb{Z}^{d},\|x-y\| \leq r$, we let $\left\{T_{n}^{x, y}: n \geq 1\right\}$ and $\left\{U_{n}^{x}: n \geq 1\right\}$ be the arrival times of Poisson processes with rates $\lambda$ and 1 , respectively. At times $T_{n}^{x, y}$, we draw an arrow from $x$ to $y$ to indicate that a birth may occur. More precisely, if $x$ is occupied and $y$ is vacant, then the particle present at site $x$ gives birth, at $y$, to a particle of the same type. At times $U_{n}^{x}$, we put a cross at $x$ to indicate that a particle of type 1 present at $x$ becomes of type 2, while a particle of type 1 is killed. A result of Harris (1972) implies that such a graphical representation can be used to construct the process starting from any initial configuration $\xi_{0}: \mathbb{Z}^{d} \rightarrow\{0,1,2\}$. See Figure 1 for a picture of the graphical representation.

Having constructed the graphical representation, we can now define the dual process. We say that $(x, 0)$ and $(y, t)$ are strongly connected, and write $(x, 0) \rightarrow(y, t)$, if there is a sequence of times $s_{0}=0<s_{1}<\cdots<s_{n}<s_{n+1}=t$, and one of spatial locations $x_{0}=x, x_{1}, \ldots, x_{n}=y$, such that the following conditions hold:

1. For $i=1,2, \ldots, n$, there is an arrow from $x_{i-1}$ to $x_{i}$ at time $s_{i}$.

2. For $i=0,1, \ldots, n$, the vertical segments $\left\{x_{i}\right\} \times\left(s_{i}, s_{i+1}\right)$ do not contain any crosses.

If, instead of condition 2 , we have the condition that

$2^{\prime}$. the set $\bigcup_{i=0}^{n}\left\{x_{i}\right\} \times\left(s_{i}, s_{i+1}\right)$ contains exactly one cross,

we say that $(x, 0)$ and $(y, t)$ are weakly connected, and write $(x, 0) \rightarrow(y, t)$. Since the crosses do not kill the type-1s but rather change them into type-2s, in the construction of the dual we must take into account the paths that contain one cross. So, to define the dual process, we reverse both the arrows and time, by mapping $\tilde{s}=t-s$, and let

$$
\tilde{\xi}_{s}^{(x, t)}=\left\{y \in \mathbb{Z}^{d}:(x, \tilde{0}) \rightarrow(y, \tilde{s}) \text { or }(x, \tilde{0}) \rightarrow(y, \tilde{s})\right\} .
$$

Since it will be easier to work with a forward process than with a backward one, we also introduce the dual $\hat{\xi}_{s}^{(x, 0)}$, which is constructed from the graphical representation that has an 
(a)

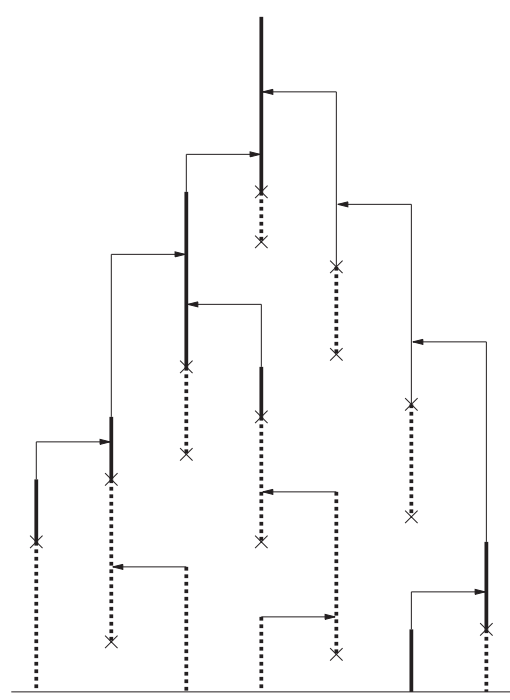

(b)

$(x, 0)$

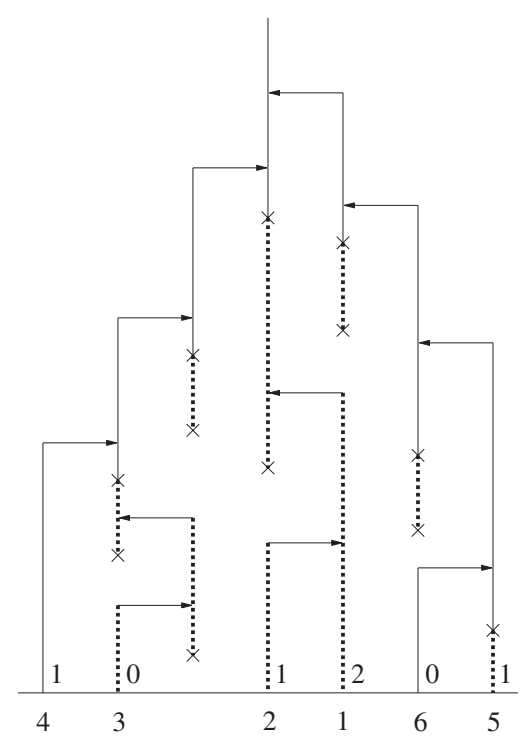

Figure 2: Pictures of the dual process. The solid lines refer to the upper tree and the dotted lines to the lower trees. In (a), the path of the distinguished particle is drawn in bold lines. Part (b) gives an example of ancestor hierarchy.

arrow from $x$ to $y$ at time $T_{n}^{x, y}$, and is defined by

$$
\hat{\xi}_{s}^{(x, 0)}=\left\{y \in \mathbb{Z}^{d}:(x, 0) \rightarrow(y, s) \text { or }(x, 0) \rightarrow(y, s)\right\} .
$$

This can be done because the processes $\tilde{\xi}_{s}^{(x, t)}$ and $\hat{\xi}_{s}^{(x, 0)}$ have the same law.

Before going into the proof of Theorem 3, we now investigate the geometry of the dual process. First of all, we observe that $\left\{\hat{\xi}_{s}^{(x, 0)}: s \geq 0\right\}$ has a tree structure consisting of the points that are either strongly or weakly connected. We first denote by $\Gamma$ the set of points strongly connected to $(x, 0)$, i.e.

$$
\Gamma=\left\{(y, t) \in \mathbb{Z}^{d} \times \mathbb{R}^{+}:(x, 0) \rightarrow(y, t)\right\} .
$$

It is clear that the tree $\Gamma$ is broken at some points by a cross, at which a new tree forms. Therefore, the picture we obtain is that of an expanding cone of trees connected to $\Gamma$ by crosses. In the following, $\Gamma$ will be called the upper tree starting at $(x, 0)$ and the trees starting at a cross the lower trees. For an illustration, see Figure 2(a), where $\Gamma$ is drawn in solid lines and the lower trees in dotted lines.

Although the dual process seems to be a complicated object, in view of the translation invariance of the graphical representation, it can be broken up into identically distributed pieces. More precisely, the upper tree and each of the lower trees have the same law as the tree structure of the contact process with parameter $\lambda$. (See, e.g. Durrett (1995) or Liggett (1999) for a construction of the contact process.) We now denote by $\hat{\xi}_{s}^{x, 1}$ and $\hat{\xi}_{s}^{x, 2}$ the dual subsets given by

$$
\hat{\xi}_{s}^{x, 1}=\left\{y \in \mathbb{Z}^{d}:(x, 0) \rightarrow(y, s)\right\} \quad \text { and } \quad \hat{\xi}_{s}^{x, 2}=\left\{y \in \mathbb{Z}^{d}:(x, 0) \rightarrow(y, s)\right\} .
$$

By analogy with the division of the tree structure into upper and lower trees, the elements of $\hat{\xi}_{s}^{x, 1}$ and $\hat{\xi}_{s}^{x, 2}$ will be called, respectively, upper ancestors and lower ancestors. As for the multitype 
contact process, the tree structure of the dual process $\left\{\hat{\xi}_{s}^{(x, 0)}: s \geq 0\right\}$ allows us to define an ancestor hierarchy in which the members are arranged according to the order in which they determine the colour of $(x, 0)$. Here, the geometry of the dual also plays an important role, since the colour of $(x, 0)$ strongly depends on the types of its ancestors. To be more precise, we now explain in greater detail how to deduce the colour of $(x, 0)$ from the hierarchy, and types, of its ancestors.

First, we denote by $\hat{\xi}_{s}^{(x, 0)}(n)$ the $n$th member of the ordered ancestor set and let $\hat{\xi}_{s}^{x, 1}(k)=$ $\hat{\xi}_{s}^{(x, 0)}\left(n_{k}\right)$ be the $k$ th upper ancestor. Later, $\hat{\xi}_{s}^{(x, 0)}(1)$ and $\hat{\xi}_{s}^{x, 1}(1)$ will be used to denote the determining particle and the distinguished particle, respectively. For a picture of the path of the distinguished particle, see Figure 2(a). We now give an algorithm to determine the colour of $(x, 0)$ depending on the type of each ancestor and the initial configuration. First of all, we look at the determining particle to establish which of the following four events occurs.

1. The ancestor is an upper ancestor that lands on a type-1.

2. The ancestor is an upper ancestor that lands on a type-2 or a lower ancestor that lands on a type-1.

3. The ancestor is a lower ancestor that lands on a type-2.

4. The ancestor lands on an empty site.

In case 1 or 2 , the determining particle will paint $(x, 0)$ the colour 1 or the colour 2 , respectively, and the algorithm halts. In cases 3 and 4 , the determining particle cannot paint $(x, 0)$ any colour. Instead, in case 4, we repeat the same reasoning with the second ancestor of the hierarchy (instead of the determining particle). In case 3, the particle of type 2 can block some other ancestors from determining the colour of $(x, 0)$. Since these ancestors can no longer determine the colour of $(x, 0)$, we must remove them from the hierarchy. To do this, we follow the determining particle on its way up to $(x, 0)$ until we first encounter a cross, then remove from the hierarchy all the ancestors of the dual process starting at this cross, and repeat the same reasoning with the first remaining ancestor of $(x, 0)$. If, after the second trial, no ancestor can paint $(x, 0)$ any colour, that is, either case 3 or 4 occurs one more time, then we start again with the next ancestor, and so on.

We now refer to Figure 2(b) for an illustration of the algorithm. The determining particle is a lower ancestor that lands on a type-2 (case 3$)$, so it cannot paint $(x, 0)$ any colour. The first cross the determining particle encounters on its way up is located at site $x$, at the top of the figure. The ancestors of this cross are the determining particle and the second ancestor, so we try again with the third ancestor. This ancestor lands on an empty site (case 4), so we look at the fourth ancestor; this is an upper ancestor that lands on a type-1 (case 1), so it paints $(x, 0)$ the colour 1 and the algorithm halts.

As we will further see, the state of $(x, 0)$ strongly depends on the spatial locations of the first ancestor and the distinguished particle. Fortunately, by using an idea of Kuczek (1989), we can easily manage the path of the distinguished particle, which is crucial to the proof of Theorem 3. To be precise, its path can be broken into independent, identically distributed pieces at certain points called renewal points. To define these points, we follow the path of the distinguished particle starting at $(x, 0)$ and, each time it jumps to a site that lives forever, call this site a renewal point. Let $\left(S_{n}, T_{n}\right)$ be the location of the $n$th renewal. We denote by $X_{i}$ the spatial displacement between consecutive renewal points and by $\tau_{i}$ the corresponding temporal displacement, so that $S_{n}=x+\sum_{i=1}^{n} X_{i}$ and $T_{n}=\sum_{i=1}^{n} \tau_{i}$. One of the main ingredients we 
need to prove Theorem 3 is then given by the following proposition. For the details of the proof, see Section 2 of Neuhauser (1992).

Proposition 1. (Neuhauser (1992).) If the upper tree lives forever, $\left\{\left(X_{i}, \tau_{i}\right)\right\}_{i \geq 1}$ form an independent, identically distributed family of random vectors on $\mathbb{Z}^{d} \times \mathbb{R}^{+}$. Moreover, we have the exponential bounds $\mathrm{P}\left(\left\|X_{i}\right\|>t\right) \leq C \mathrm{e}^{-\beta t}$ and $\mathrm{P}\left(\tau_{i}>t\right) \leq C \mathrm{e}^{-\beta t}$ for appropriate $C<\infty$ and $\beta>0$.

In conclusion, Proposition 1 gives us control over the location of the distinguished particle at the renewal points. Moreover, the contact process grows at most linearly in space - see, e.g. Section 1 of Durrett (1988) - so, between consecutive renewals, the particle stays within a linearly growing set that we will refer to as a triangle, henceforth.

\section{Proof of Theorem 3 in dimension less than or equal to two}

The techniques and tools we will use to prove Theorem 3 differ considerably, depending on the dimension of the state space. In this section, we deal with the case $d \leq 2$, relying on the recurrence of one- and two-dimensional random walks. To begin with, we will show that the determining particle is trapped with probability 1 inside a lower tree that lives forever, so that, for $t$ large enough, the determining particle is a lower ancestor. At this point, the worst scenario we have in mind is that the distinguished particle lands on a type- 1 and the determining particle on a type-2. In such a case, the determining particle, which is a lower ancestor, cannot paint $(x, 0)$ any colour, whereas the distinguished particle can possibly bring a type-1 to $(x, 0)$. To conclude, we will then prove that this undesirable event is negligible, showing that, with probability 1 , we can make the distinguished particle and the determining particle coalesce, and so make them land on the same site. If this site is occupied by a particle of type 1 , the determining particle will paint $(x, 0)$ the colour 2 . If the site is occupied by a particle of type 2 , the distinguished particle will paint $(x, 0)$ the colour 2 , unless a lower ancestor does so earlier.

First, we can observe that if the upper tree starting at $(x, 0)$ does not live forever, then, for $t$ large enough, $\hat{\xi}_{t}^{(x, 0)}=\hat{\xi}_{t}^{x, 2}$. In particular, since each of the lower ancestors meets one cross, the point $(x, 0)$ cannot be reached in this case by a type-1 (see the description of the ancestor hierarchy in Section 3). So, to avoid trivialities, we suppose that the upper tree $\Gamma$ lives forever. Note that the probability of such an event is equal to the survival probability of the contact process with parameter $\lambda$ starting from one infected site, which is positive since $\lambda>\lambda_{\mathrm{c}}$. The first step is to prove that the determining particle is almost surely trapped inside a lower tree after a finite time. More precisely, if we let $\Theta_{1}$ be the last time the determining particle is strongly connected with $(x, 0)$, then the following lemma holds.

Lemma 2. $\mathrm{P}\left(\Theta_{1}=\infty\right)=0$.

Proof. Let $\left\{s_{k}: k \geq 1\right\}$ be the jumping times of the determining particle and $x_{k}$ its location before $s_{k}$. Denote by $\sigma_{1}$ the first time the particle encounters a cross, i.e.

$$
\sigma_{1}=\inf \left\{t \geq 0: \hat{\xi}_{t}^{(x, 0)}(1) \text { is a lower ancestor }\right\}
$$

and by $\Omega_{1}$ the lower tree starting at $\left(x_{1}, \sigma_{1}\right)$ : that is, the first lower tree the particle visits - see the left-hand diagram of Figure 3 for an illustration. In view of the duality properties, once the particle enters $\Omega_{1}$, it remains trapped inside (as long as the lower tree is alive). Hence, if $\Omega_{1}$ lives forever, $\hat{\xi}_{t}^{(x, 0)}(1)$ is a lower ancestor for any $t \geq \sigma_{1}$, and the proof is done. Otherwise, we 

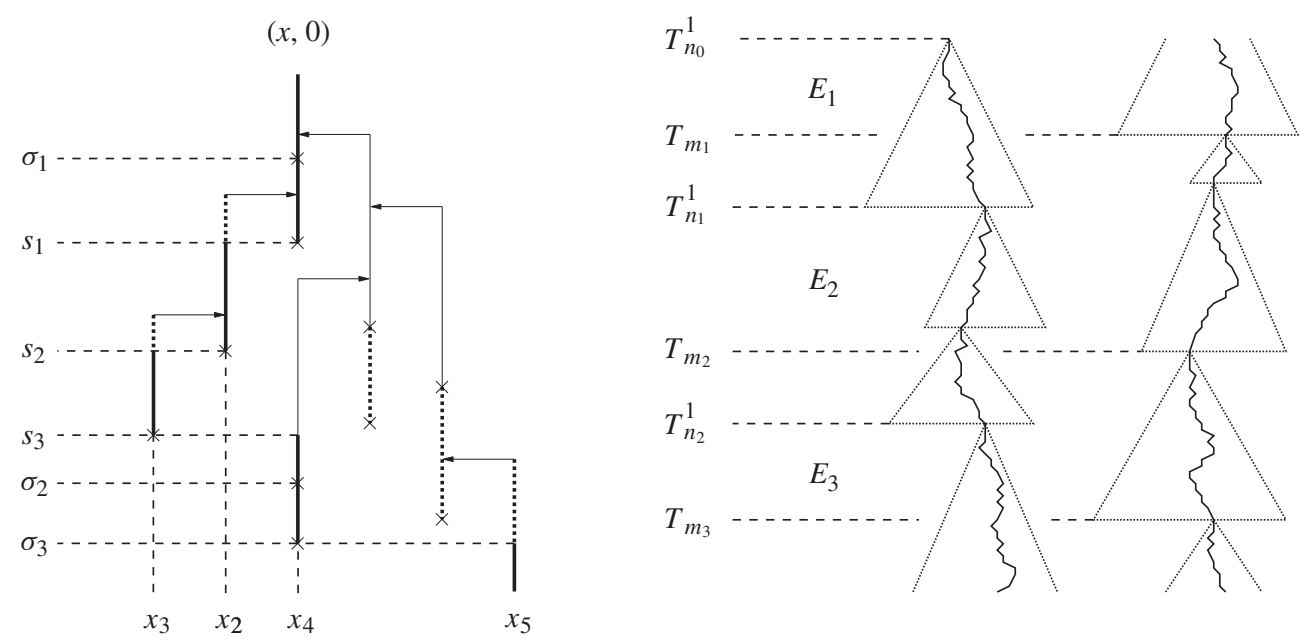

Figure 3: Pictures of the dual process.

denote by $\sigma_{2}$ the first time the determining particle visits a new lower tree after $\Omega_{1}$ dies, and by $\Omega_{2}$ this lower tree. Note that, for all $k \geq 1$, the path the particle takes to climb from $\left(x_{k}, s_{k}^{-}\right)$ to $(x, 0)$ contains one cross, so $\sigma_{2}$ is almost surely finite and $\Omega_{2}$ is well defined. In this way, while the particle is not trapped in a lower tree that lives forever, we construct by induction a sequence of trees $\Omega_{k}$ visited by the first ancestor.

Now denote by $B_{n}$ the event that the first $n$ trees $\Omega_{1}, \Omega_{2}, \ldots, \Omega_{n}$ are bounded and, by $A_{k}$ for any $k \geq 1$, the event that the $k$ th tree lives forever. If $A_{k}$ does not occur then $\Omega_{k+1}$ is well defined and the event $A_{k+1}$ is determined by parts of the graph that are after the death of $\Omega_{k}$, so $A_{k}$ and $A_{k+1}$ are independent. More generally, since the trees $\Omega_{1}, \Omega_{2}, \ldots, \Omega_{k+1}$ are disjoint, $A_{1}, A_{2}, \ldots, A_{k+1}$ are independent. Moreover, since $\Omega_{k}$ has the same distribution as the tree structure of the contact process, the probability that $A_{k}$ occurs is given by $p_{\lambda}$, the survival probability of the contact process with parameter $\lambda$ starting from one infected site. This implies that

$$
\mathrm{P}\left(B_{n}\right)=\mathrm{P}\left(A_{1}^{\mathrm{c}} \cap \cdots \cap A_{n-1}^{\mathrm{c}} \cap A_{n}^{\mathrm{c}}\right)=\prod_{k=1}^{n} \mathrm{P}\left(A_{k}^{\mathrm{c}}\right)=\left(1-p_{\lambda}\right)^{n},
$$

where the superscript ' $c$ ' denotes complement. Finally, since $\lambda$ is supercritical, the survival probability of the contact process $p_{\lambda}$ is strictly positive, so that $\lim _{n \rightarrow \infty} \mathrm{P}\left(B_{n}\right)=0$. This completes the proof of the lemma.

The next step is to prove that the determining and distinguished particles coalesce with probability 1 . To do this, we first note that, after entering a lower tree $\Gamma_{1}$ that never dies, the determining particle is weakly connected to $(x, 0)$ and so jumps to a new branch each time it meets a cross. In particular, from time $\Theta_{1}$, the path of the determining particle can be broken up, as can that of the distinguished particle, into independent, identically distributed pieces. We define the renewal points of the determining particle as before, replacing the upper tree $\Gamma$ by the lower tree $\Gamma_{1}$ starting at $\left(S_{0}^{1}, \Theta_{1}\right)$. Here, $S_{0}^{1}$ is the site to which the determining particle jumps at time $\Theta_{1}$. We denote by $\left(S_{n}^{1}, T_{n}^{1}\right)$ the location of the $n$th renewal after $\Theta_{1}$, and by $X_{i}^{1}$ 
and $\tau_{i}^{1}$ the spatial and temporal displacements between two consecutive renewals, so that

$$
S_{n}^{1}=S_{0}^{1}+\sum_{i=1}^{n} X_{i}^{1} \quad \text { and } \quad T_{n}^{1}=\Theta_{1}+\sum_{i=1}^{n} \tau_{i}^{1} .
$$

By translation invariance of the graphical representation (see the description of the tree structure in Section 3), the families $\left\{\left(X_{i}, \tau_{i}\right)\right\}_{i \geq 1}$ and $\left\{\left(X_{i}^{1}, \tau_{i}^{1}\right)\right\}_{i \geq 1}$ are identically distributed, so Proposition 1 holds for the random vectors $\left(X_{i}^{1}, \tau_{i}^{1}\right)$ also. In particular, as long as their triangles do not collide, both particles behave nearly like independent random walks. This constitutes the main ingredient in the proof of coalescence.

The first step in establishing coalescence is to extend the notion of renewals for both particles, that is, to break up the set of both paths into independent, identically distributed pieces. To do so, we say that an ancestor is good at time $t$ if it has not met any arrows since its last renewal. Observe that if both particles are good at the same time, the events before and after that time use disjoint parts of the graph and so are independent. We can now present the following lemma.

Lemma 3. Both particles are simultaneously good infinitely often, i.e.

$\mathrm{P}($ the determining and distinguished particles are simultaneously good infinitely often $)=1$.

Proof. By induction, we first construct two sequences of subscripts $\left(n_{k}\right)_{k \geq 0}$ and $\left(m_{k}\right)_{k \geq 1}$, as follows. We let $n_{0}=1$ and, for any $k \geq 1$, we let

$$
m_{k}=\min \left\{m \geq 1: T_{m}>T_{n_{k-1}}^{1}\right\} \quad \text { and } \quad n_{k}=\min \left\{n \geq 1: T_{n}^{1}>T_{m_{k}}\right\} .
$$

Then denote by $E_{k}$ the event that the determining particle lives, without giving birth, between time $T_{n_{k-1}}^{1}$ and time $T_{m_{k}}$ - see the right-hand diagram of Figure 3 for an illustration. Note that if $E_{k}$ occurs, both particles are obviously good at time $T_{m_{k}}$. Moreover, in view of the exponential bound given by Proposition 1, for any time $T>0$,

$$
\mathrm{P}\left(T_{m_{k}}-T_{n_{k-1}}^{1}>T\right) \leq \mathrm{P}\left(T_{m_{k}}-T_{m_{k}-1}>T\right)=\mathrm{P}\left(\tau_{1}>T\right) \leq C \mathrm{e}^{-\beta T},
$$

so that $\mathrm{P}\left(E_{k}\right) \geq\left(1-C \mathrm{e}^{-\beta T}\right) \mathrm{e}^{-(1+2 d \lambda) T}$. Here, $\mathrm{e}^{-(1+2 d \lambda) T}$ is the probability that the determining particle lives, without giving birth, for $T$ units of time. Since this holds for all $T>0$, there exists a constant $\varepsilon_{0}>0$ such that $\mathrm{P}\left(E_{k}\right) \geq \varepsilon_{0}$. In other respects, the events $E_{k}$ are determined by disjoint parts of the graphical representation, so they are independent. Hence, by the Borel-Cantelli lemma, we can conclude that

$\mathrm{P}($ both particles are simultaneously good infinitely often $) \geq \mathrm{P}\left(\limsup _{k \rightarrow \infty} E_{k}\right)=1$.

This proves the lemma.

Now, to cause the particles to coalesce, we proceed in two steps. First, relying on the recurrence of one- and two-dimensional random walks, Neuhauser (1992) proved that, with positive probability, we can bring both particles to within a finite distance $K$ of one another without intersection of their triangles. Then, as soon as the particles are close enough to each other, we try to make them coalesce. More precisely, we have the following lemma.

Lemma 4. If the determining and distinguished particles are within a distance $K$ of one another at some time $t \geq \Theta_{1}$ then the event $A$, that they coalesce, has positive probability. 
Proof. To find a lower bound for $\mathrm{P}(A)$, we consider the following particular event: we require the distinguished particle to keep still for $3 d K$ units of time and the determining particle to jump towards it until they coalesce. To estimate the probability of this event, we observe that, since the ancestors are within a distance $K$ of one another, it takes the determining particle at most $d K$ steps by increasing or decreasing each of its coordinates to reach the distinguished particle. Moreover, the probability of neither a birth nor a death occurring between times 0 and 1 , forming a good oriented arrow between times 1 and 2, and a death occurring between times 2 and 3 is given by

$$
\mathrm{e}^{-2}\left(1-\mathrm{e}^{-1}\right) \mathrm{e}^{-(4 d-1) \lambda}\left(1-\mathrm{e}^{-\lambda}\right)
$$

Since it takes the determining particle at most $3 d K$ units of time to reach the distinguished particle in the manner just described, we can conclude that

$$
\mathrm{P}(A) \geq \mathrm{e}^{-3 d K(1+2 d \lambda)}\left[\mathrm{e}^{-2}\left(1-\mathrm{e}^{-1}\right) \mathrm{e}^{-(4 d-1) \lambda}\left(1-\mathrm{e}^{-\lambda}\right)\right]^{d K}>0,
$$

where $\mathrm{e}^{-3 d K(1+2 d \lambda)}$ is the probability that the distinguished particle survives without giving birth for $3 d K$ units of time. This completes the proof.

If we do not succeed in 'gluing' the particles together, i.e. causing them to coalesce, we use the restart argument given by Lemma 3, i.e. we wait until both particles are good at the same time and then start the whole procedure over again. Since the set of both paths is broken into independent, identically distributed pieces, we can apply the Borel-Cantelli lemma to conclude that coalescence eventually occurs.

To complete the proof of Theorem 3 in $d \leq 2$ we now use the dual process $\tilde{\xi}_{s}^{(x, t)}, 0 \leq$ $s \leq t$, starting at $(x, t)$, and determine the ancestor hierarchy after $t$ units of time by evolving backwards in time. First of all, since the particles coalesce almost surely, we can suppose, by taking $t$ large enough, that they land on the same site at time 0 . If this site is occupied by a type-1, the determining particle, which is a lower ancestor (by Lemma 2 ), will paint $(x, t)$ the colour 2. On the other hand, if both land on a type-2, it is the distinguished particle that will paint $(x, t)$ the colour 2, unless a lower ancestor succeeds in doing so earlier. Finally, if the target site is empty, we start over again with the second ancestor, and so on. Since the tree starting at the point where the particles coalesce is growing linearly in time, and since $\xi_{0}$ is translation invariant, we eventually find, by Lemma 9.14 of Harris (1976), an ancestor landing on an occupied site that will bring a type- 2 to $(x, t)$. This concludes the proof.

\section{Proof of Theorem 3 in dimension greater than or equal to three}

The strategy of the proof to deal with the case $d \geq 3$ is quite different. To begin with, we will construct, by induction, an ordered set of ancestors $\zeta_{t}(k), k \geq 1$, that are candidates for painting $(x, 0)$ the colour 2. Using Lemma 2, we will prove that for any $k \geq 1$, and for $t$ sufficiently large, $\zeta_{t}(k)$ is a lower ancestor that comes before the distinguished particle in the ancestor hierarchy. Then, relying on the transience of $d$-dimensional random walks for $d \geq 3$, we will extract a subsequence of ancestors, denoted by $\zeta_{t}\left(k_{i}\right), i \geq 1$, that never coalesce. In particular, the number of sites occupied by these candidates can be made arbitrarily large, so that we find one landing on a type-1, which will paint $(x, 0)$ the colour 2 .

We start by inductively constructing the ordered ancestor set $\zeta_{t}(k), k \geq 1$. The first member of the sequence $\zeta_{t}(1)$ is the determining particle. Before defining the second member, we wait until the first one enters a lower tree $\Gamma_{1}$ that lives forever. By Lemma 2, the time $\Theta_{1}$ when this event occurs is almost surely finite, so $\Gamma_{1}$ is well defined. Then we look at the ancestor 
hierarchy at time $\Theta_{1}$ and discard all the ancestors that land either on $S_{0}^{1}$ or on a site that does not live forever. Here, $S_{0}^{1}$ is the spatial location of the determining particle at time $\Theta_{1}$. The second member of the sequence is then the first remaining ancestor. Observe that such an ancestor exists, since we assumed that $\Gamma$ lives forever. Moreover, by using the arguments of Lemma 2, one can prove that $\zeta_{t}(2)$ also enters, with probability 1 , a lower tree $\Gamma_{2}$ that lives forever. We then repeat the procedure in order to define the third candidate, and so on.

For any $k \geq 1$, we let $\Gamma_{k}$ be the infinite lower tree visited by the $k$ th member of the sequence, and denote by $\Theta_{k}$ the first time the ancestor enters $\Gamma_{k}$ and by $S_{0}^{k}$ its spatial location at time $\Theta_{k}$. As before, from time $\Theta_{k}$, we can break up the path of the $k$ th ancestor into independent, identically distributed pieces. We denote by $\left(S_{n}^{k}, T_{n}^{k}\right)$ the $n$th renewal point, by $X_{i}^{k}$ the spatial displacement between consecutive renewals, and by $\tau_{i}^{k}$ the corresponding temporal displacement, i.e.

$$
S_{n}^{k}=S_{0}^{k}+\sum_{i=1}^{n} X_{i}^{k} \quad \text { and } \quad T_{n}^{k}=\Theta_{k}+\sum_{i=1}^{n} \tau_{i}^{k} .
$$

Our strategy to prove that, with probability 1 , there exists a subsequence $\zeta_{t}\left(k_{i}\right)$ of lower ancestors that never coalesce, is the following. First of all, we let $n \in \mathbb{N}^{+}$, the set of positive integers, and assume that there exist $n$ lower ancestors $\zeta_{t}\left(k_{1}\right), \zeta_{t}\left(k_{2}\right), \ldots, \zeta_{t}\left(k_{n}\right)$ that never coalesce. Then, given $m>k_{n}$, we will prove that the event $G_{m, K}$, that we can separate $\zeta_{t}(m)$ by a distance of at least $K$ units from each of the $\zeta_{t}\left(k_{i}\right)$ and then trap the $n+1$ ancestors inside large, disjoint cubes without intersection of their triangles, has positive probability. (Here, ' $G$ ' signifies a good event.) Then, relying on Lemma 5.5 of Neuhauser (1992), we will prove that in the event $G_{m, K}$, and for $K$ sufficiently large,

$$
\lim _{t \rightarrow \infty} \inf _{1 \leq i \leq n}\left\|\zeta_{t}\left(k_{i}\right)-\zeta_{t}(m)\right\|=\infty
$$

with probability close to 1 . We will then conclude by proving that, with probability 1 , the good events $G_{m, K}$ occur for infinitely many $m>k_{n}$. In what follows, we give the proofs for the determining particle only, but the same arguments hold for the other members of the sequence.

Lemma 5. Let $K \in \mathbb{N}^{+}$and $D_{K}=[-K, K]^{d}$. For any $t \geq \Theta_{1}$ the event $B$, that the determining particle leaves $D_{K}$ in less than one unit of time and then survives without giving birth until time $t+K$, has positive probability.

Proof. If the determining particle leaves $D_{K}$ at time $t$, then $\mathrm{P}(B)$ can be bounded from below by $\mathrm{e}^{-(1+2 d \lambda) K}$, that is, the probability of living without giving birth for $K$ units of time. Otherwise, we require the first ancestor to increase or decrease its first spatial coordinate until leaving $D_{K}$. A straightforward calculation shows that

$$
\mathrm{P}(B) \geq \mathrm{e}^{-(1+2 d \lambda) K}\left[\mathrm{e}^{-1 / K}\left(1-\mathrm{e}^{-1 / 2 K}\right) \mathrm{e}^{-d \lambda / K}\left(1-\mathrm{e}^{-d \lambda / K}\right)\right]^{K}>0,
$$

since it takes at most $K$ steps for the ancestor to leave $D_{K}$. This concludes the proof.

Lemma 6. Let $H_{t, K}$ be the event that the triangles of the first ancestor are inside a spatial box $z+D_{K}$ from time t to time $t+K$. Then, for any $\varepsilon_{1}>0$, there exists $K$ such that $\mathrm{P}\left(H_{t, K}\right) \geq 1-\varepsilon_{1}$.

Proof. The proof is, with minor modifications, the same as that of Lemma 4.11 in Neuhauser (1992). 
We are now ready to prove that, with probability 1 , there is an integer $m>k_{n}$ such that $\zeta_{t}(m)$ does not coalesce with the first $n$ members of the subsequence $\zeta_{t}\left(k_{i}\right)$. We let $G_{m, K}$ now be the event that, for any $1 \leq i \leq n$, there is a time $t_{i} \in\left[\Theta_{m}, \Theta_{m}+K\right]$ such that $\zeta_{t}\left(k_{i}\right)$ and $\zeta_{t}(m)$ are good and at least $\bar{K}$ units apart from each other at time $t=t_{i}$.

Lemma 7. For any $\varepsilon_{2}>0$, there exists $K$ sufficiently large that, in the event $G_{m, K}$,

$$
\mathrm{P}\left(\lim _{t \rightarrow \infty} \inf _{1 \leq i \leq n}\left\|\zeta_{t}\left(k_{i}\right)-\zeta_{t}(m)\right\|=+\infty\right) \geq 1-\varepsilon_{2} .
$$

Proof. We let $1 \leq i \leq n$ and assume that the ancestors $\zeta_{t}\left(k_{i}\right)$ and $\zeta_{t}(m)$ are good and $K$ units apart at some time $t \geq \Theta_{m}$. Then, Lemma 5.5 of Neuhauser (1992) implies that there exists a constant $C>0$ such that

$$
\begin{aligned}
\mathrm{P}\left(\text { the particles } \zeta_{t}\left(k_{i}\right) \text { and } \zeta_{t}(m) \text { never coalesce }\right) & =\mathrm{P}\left(\lim _{t \rightarrow \infty}\left\|\zeta_{t}\left(k_{i}\right)-\zeta_{t}(m)\right\|=+\infty\right) \\
& \geq 1-C K^{-1 / 10}-2 C K^{-3 / 32} .
\end{aligned}
$$

In particular, for $K$ large enough,

$$
\begin{aligned}
\mathrm{P}\left(\zeta_{t}\left(k_{i}\right) \text { and } \zeta_{t}(m) \text { coalesce for some } 1 \leq i \leq n\right) & \leq \sum_{i=1}^{n} \mathrm{P}\left(\zeta_{t}\left(k_{i}\right) \text { and } \zeta_{t}(m) \text { coalesce }\right) \\
& \leq n C K^{-1 / 10}+2 n C K^{-3 / 32} \\
& \leq \varepsilon_{2}
\end{aligned}
$$

This proves the lemma.

Lemma 8. There exists $K$ sufficiently large that $\mathrm{P}\left(\lim \sup _{m \rightarrow \infty} G_{m, K}\right)=1$.

Proof. Since the $n$ lower ancestors $\zeta_{t}\left(k_{i}\right)$ do not coalesce, and $\Theta_{m} \rightarrow \infty$, we can find, by Lemma 7, an $m$ large enough that the distances between the ancestors at time $\Theta_{m}$ are bigger than $4 d^{1 / 2} K$. We denote by $H_{i}$ the event that the triangles of $\zeta_{t}\left(k_{i}\right)$ are contained, between time $\Theta_{m}$ and time $\Theta_{m}+K$, in some box $\Omega_{i}=z_{i}+D_{K}$. Observe that $m$ has been chosen so that $\Omega_{i} \cap \Omega_{j}=\varnothing$ for $i \neq j$. Hence, the events $H_{i}$ are determined by disjoint parts of the graph and, so, are independent. This, together with Lemma 6, implies that, for $K$ sufficiently large,

$$
\mathrm{P}\left(H_{1} \cap \cdots \cap H_{n}\right)=\prod_{i=1}^{n} \mathrm{P}\left(H_{i}\right) \geq 1-n \varepsilon_{1} .
$$

Now that the $n$ lower ancestors $\zeta_{t}\left(k_{i}\right)$ are trapped inside large disjoint cubes, we require each of them to be good at least once between time $\Theta_{m}$ and time $\Theta_{m}+K$. Since this occurs if each of the $n$ ancestors has at least one renewal in this time interval, the probability that this event occurs can be bounded from below by

$$
\prod_{i=1}^{n} \mathrm{P}\left(\tau_{1}^{k_{i}}<K\right) \geq\left[1-C \mathrm{e}^{-\beta K}\right]^{n},
$$

for appropriate $C<\infty$ and $\beta>0$. To conclude, the last fact we need to establish is that $\zeta_{t}(m)$ is good and at least $K$ units apart from each other ancestor between time $\Theta_{m}+1$ and time $\Theta_{m}+K$. Since the cubes $\Omega_{i}$ are at least $2 K$ units apart from each other, this occurs, by 
Lemma 5, with positive probability. Putting things together, we can state that there exists an $\varepsilon_{3}>0$ such that $\mathrm{P}\left(G_{m, K}\right) \geq \varepsilon_{3}$ for $m$ sufficiently large. By observing that the events $G_{m_{1}, K}$ and $G_{m_{2}, K}$ are independent for $\left|\Theta_{m_{1}}-\Theta_{m_{2}}\right|>K$, we can conclude, by the Borel-Cantelli lemma, that the events $G_{m, K}$ occur with probability 1 for infinitely many $m \geq 1$.

To complete the proof of Theorem 3, we now use, as in Section 4, the dual process $\tilde{\xi}_{s}^{(x, t)}$ starting at $(x, t)$ and determine the ancestor hierarchy by evolving backwards in time. We denote by $B_{s}^{(x, t)}$ the set of sites occupied at time $s$ by a type- 1 and let $\zeta_{t}=\left\{\zeta_{t}(k): k \geq 1\right.$ with $\left.\Theta_{k} \leq t\right\}$. Lemma 7 implies that, in the event $G_{m, K}$, the lower ancestor $\zeta_{t}(m)$ coalesces with one of the $\zeta_{t}\left(k_{i}\right)$ with probability less than $\varepsilon_{2}$. Moreover, by Lemma $8, G_{m, K}$ occurs for infinitely many $m \geq 1$. This implies, in particular, that the cardinality of $\zeta_{t}$ can be made arbitrarily large by choosing $t$ large enough, i.e. given $\varepsilon_{4}>0$ and $M>0$, there exists a time $t_{0} \geq 0$ such that $\mathrm{P}\left(\operatorname{card}\left(\zeta_{t}\right)<M\right) \leq \varepsilon_{4}$ for any $t \geq t_{0}$. By Lemma 9.14 of Harris (1976), this, together with the translation invariance of $\xi_{0}$, implies that

$$
\lim _{t \rightarrow \infty} \mathrm{P}\left(\zeta_{t-1} \cap B_{1}^{(x, t)}=\varnothing\right)=0 .
$$

So, for $t$ sufficiently large, there is a lower ancestor $\zeta_{t}(n)$ that lands at time 0 on a type- 1 . Now we look at the ancestors that come before $\zeta_{t}(2)$ in the hierarchy until we find one that lands on an occupied site. If a type- 1 stands at this site, the ancestor will paint $(x, t)$ the colour 2 and the proof is done. On the other hand, if the site is occupied by a type- $2, S_{0}^{1}$ will be empty at time $\left(t-\Theta_{1}\right)^{+}$regardless of the colour of the next ancestors. Then, we look at $\zeta_{t}(2)$, and so on. Finally, if none of the ancestors that come before $\zeta_{t}(n)$ in the hierarchy succeed in painting $(x, t)$ the colour 2, this last one will do so. This completes the proof of Theorem 3.

\section{Acknowledgements}

I would like to thank Claudio Landim for suggesting the problem and Claudia Neuhauser for her advice. I am also grateful to Olivier Benois, Roberto Fernández, and Pierre Margerie for the time they devoted to me, and fruitful discussions.

\section{References}

Durrett, R. (1984). Oriented percolation in two dimensions. Ann. Prob. 12, 999-1040.

Durrett, R. (1988). Lecture Notes on Particle Systems and Percolation. Wadsworth and Brooks, Pacific Grove, CA.

Durrett, R. (1995). Ten lectures on particle systems. In Lectures on Probability Theory (Saint-Flour, 1993; Lecture Notes Math. 1608), Springer, Berlin, pp. 97-201.

Durrett, R. And Neuhauser, C. (1991). Epidemics with recovery in $d=2$. Ann. Appl. Prob. 1, 189-206.

Durrett, R. And Neuhauser, C. (1997). Coexistence results for some competition models. Ann. Appl. Prob. 7, 10-45.

HARris, T. E. (1972). Nearest-neighbor Markov interaction processes on multidimensional lattices. Adv. Math. 9, 66-89.

Harris, T. E. (1976). On a class of set-valued Markov processes. Ann. Prob. 4, 175-194.

KuczeK, T. (1989). The central limit theorem for the right edge of supercritical oriented percolation. Ann. Prob. 17, $1322-1332$.

Liggett, T. M. (1999). Stochastic Interacting Systems: Contact, Voter and Exclusion Processes. Springer, Berlin.

Neuhauser, C. (1992). Ergodic theorems for the multitype contact process. Prob. Theory Relat. Fields 91, 467-506. 